\title{
Colonoscopic extraction of a chicken wishbone stuck in the sigmoid colon, identified as divertic- ulitis: the patient's and doctor's wish comes true
}

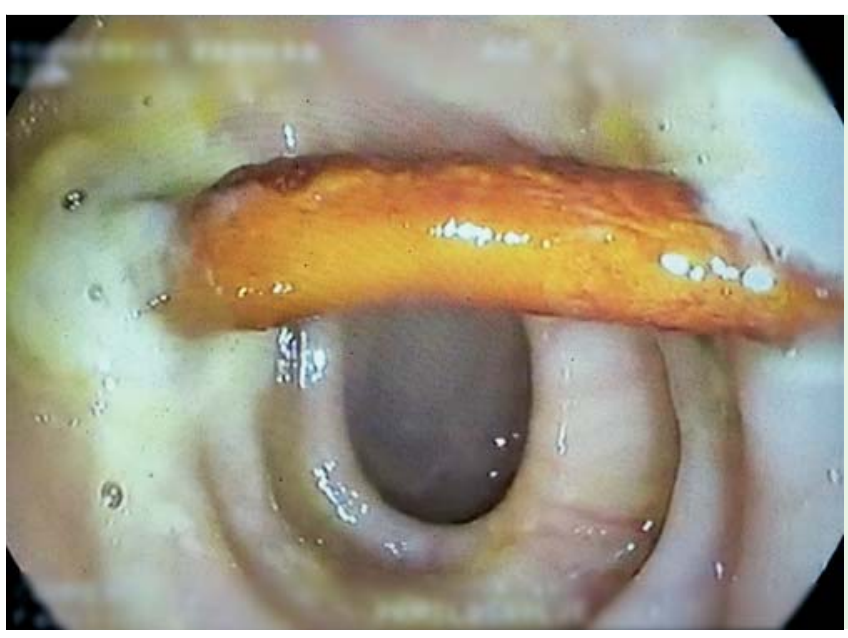

Fig. 1 A foreign body stuck in the colon wall, with one end impacted within a diverticulum and the other in the wall opposite, in a 75-year-old woman.

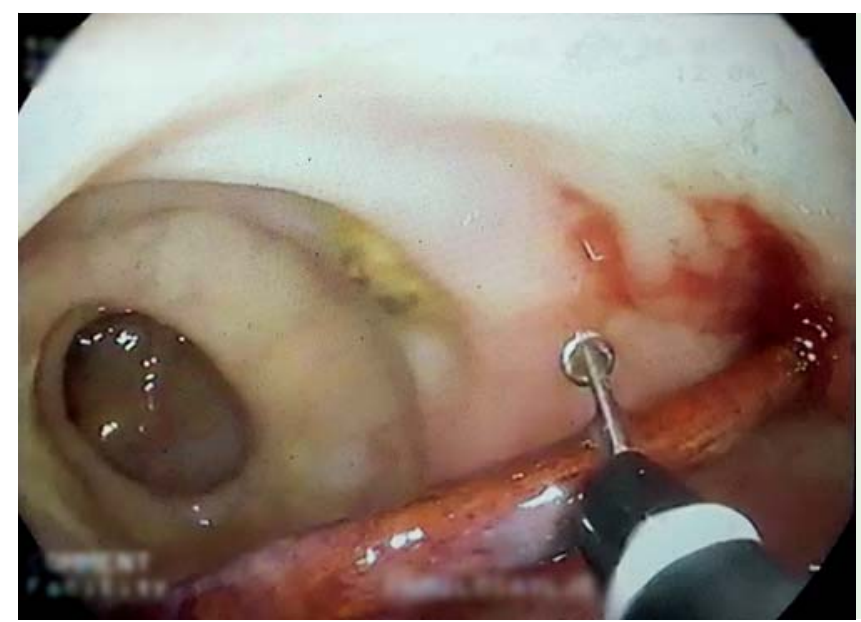

Fig. 2 The mucosa surrounding the foreign body, showing inflammation and edema.

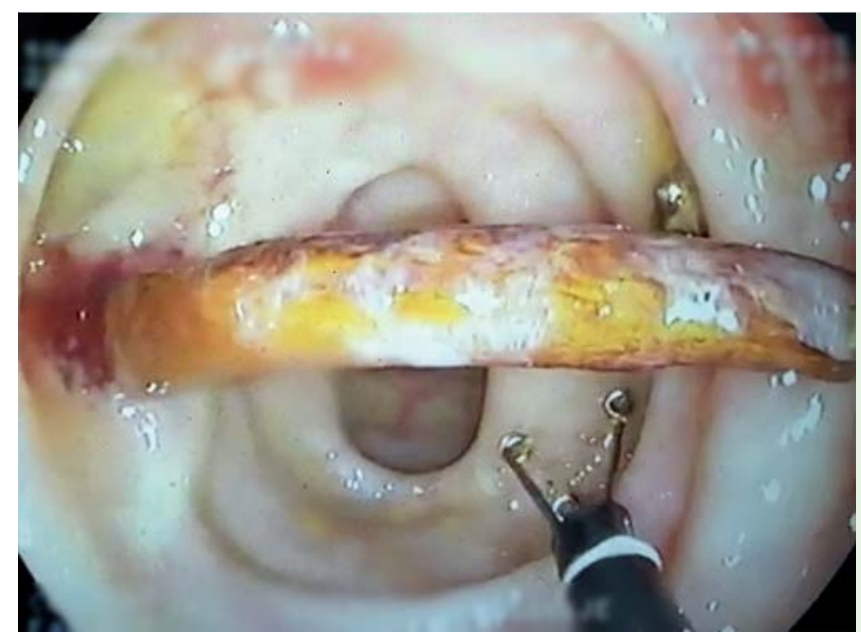

Fig. 3 The foreign body was successfully removed endoscopically in two stages using a tripod grasper.

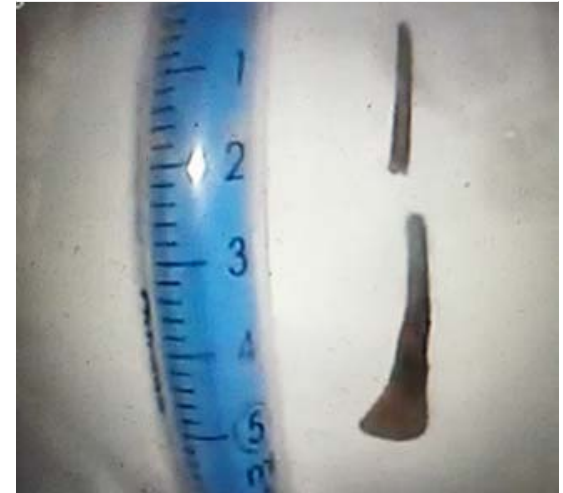

Fig.4 A 5-cm long wishbone successfully removed.

A 75-year-old woman attended our clinic presenting with a 7-day history of nausea, left lower quadrant abdominal pain, and fever. Laboratory data revealed leukocytosis (14500 white blood cells/mL) and an elevated C-reactive protein level $(28 \mathrm{mg} / \mathrm{L})$. Palpable abdominal pain in the left lower quadrant and a raised temperature $\left(38^{\circ} \mathrm{C}\right)$ were found on physical examination. Findings were negative for melena, blood per rectum, weight loss, and change in stool frequency. An abdominal X-ray showed bowel distension with one significant air-fluid level, without pneumoperitoneum. Besides renal calculosis the abdominal ultrasonography was unremarkable. Based on the clinical examination and laboratory findings, the patient's initial diagnosis was diverticulitis. A course of broad-spectrum antibiotic therapy was initiated.

After clinical and laboratory test improvement she was referred for colonoscopy, which revealed the extent of colon diverticulosis and ruled out a malignancy. An obstructing foreign body was seen in the sigmoid colon, stuck in the bowel wall ( $\bullet$ Fig. 1 and $\bullet$ Fig. 2). The foreign body was successfully removed using a tripod grasper ( $\bullet$ Fig.3 and $\odot$ Fig.4; $\diamond$ Video 1 ). The intervention was completed without any complications ( $\bullet$ Fig.5). Recovery was smooth and the patient was discharged from hospital. 

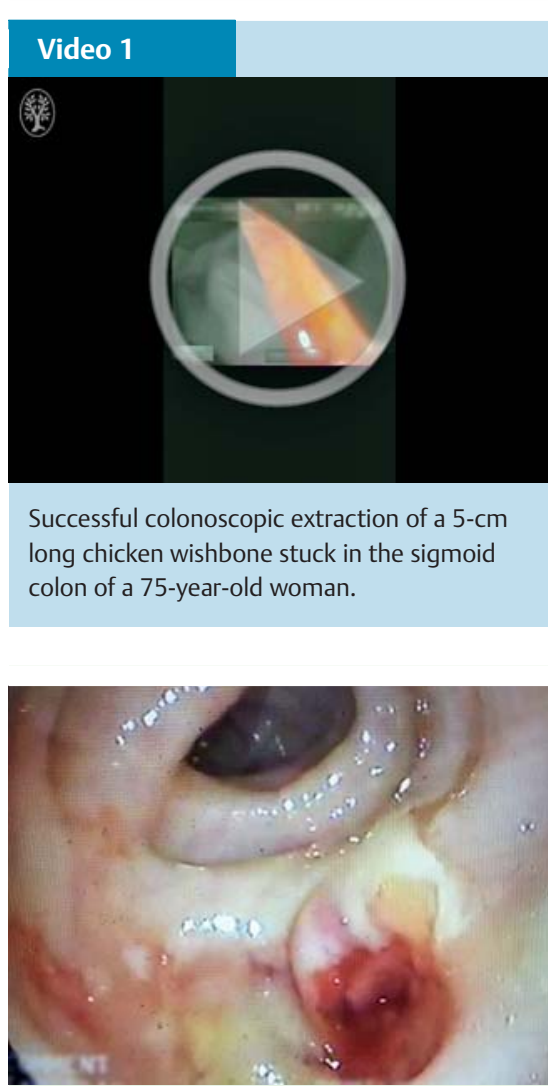

Fig. 5 The intervention was completed without any complications.
About $80 \%$ of ingested objects entering the stomach will be passed without complications [1]. Large numbers of reports support the fact that perforation is more common with sharp or pointed objects, such as chicken or fish bones, metal objects, and wooden splinters [2]. Potential sites where a foreign body tends to perforate the gastrointestinal tract are the ileocecal valve and the rectosigmoid junction [3]. In our patient, fortunately, there was no perforation, although it was threatened. Rex \& Bilotta [4] described successful colonoscopic retrieval of wishbones stuck in the sigmoid colon, without peritoneal signs, in two patients. Tarnasky et al. provided another report of an endoscopically removed chicken bone [5].

Based on the literature of which we are aware, it is rational to attempt colonoscopic removal of wishbones impacted in the sigmoid colon in patients without peritoneal findings.

Endoscopy_UCTN_Code_CCL_1AD_2AG

Competing interests: None

Vladimir Milivojevic ${ }^{1}$, Ivan Rankovic ${ }^{1}$, Bojan Djukic ${ }^{2}$, Miodrag Krstic ${ }^{1,3}$, Tomica Milosavljevic ${ }^{1,3}$

${ }^{1}$ Clinic for Gastroenterology and Hepatology, Clinical Center of Serbia, Belgrade, Serbia

${ }^{2}$ General Hospital Paracin, Paracin, Serbia

${ }^{3}$ School of Medicine, Belgrade University, Belgrade, Serbia

\section{References}

1 Henderson CT, Engel J, Schlesinger P. Foreign body ingestion: review and suggested guidelines for management. Endoscopy 1987; 19: $68-71$

2 Reddy SK, Griffith GS, Goldstein JA et al. Toothpick impaction with localized sigmoid perforation: successful colonoscopic management. Gastrointest Endosc 1999; 50: 708-709

3 Vardaki E, Maniatis V, Chrisikopoulos $\mathrm{H}$ et al. Sigmoid carcinoma incidentally discovered after perforation caused by an ingested chicken bone. AJR Am J Roentgenol 2001; 176: $153-158$

4 Rex DK, Bilotta J. Colonoscopic removal of chicken bones impacted in the sigmoid in two patients. Gastrointest Endosc 1997; 46: 193-195

5 Tarnasky PR, Newcomer MK, Branch MS. Colonoscopic diagnosis and treatment of chronic chicken bone perforation of the sigmoid colon. Gastrointest Endosc 1994; 40: $373-375$

Bibliography

DOI http://dx.doi.org/

10.1055/s-0042-120264

Endoscopy 2016; 48: E374-E375

(c) Georg Thieme Verlag KG

Stuttgart · New York

ISSN 0013-726X

Corresponding author

\section{Vladimir Milivojevic, MD}

Clinic for Gastroenterology and Hepatology

Clinical Center of Serbia

Koste Todorovića 2

Belgrade 11000

Serbia

Fax: +381-11-3662761

dotorevlada@gmail.com 